\title{
Patient with Poor Esthetic Anterior Tooth Position Treated with Lithium Disilicate Mesial Cantilever Single-retainer Fixed Partial Denture: A Case Report
}

\author{
Syuntaro Nomoto ${ }^{1)}$, Akina Sugiuchi ${ }^{2)}$, Tomohiro Asai ${ }^{2)}$, Toru Sato ${ }^{3)}$ and \\ Hideshi Sekine ${ }^{1)}$ \\ ${ }^{1)}$ Department of Fixed Prosthodontics, Tokyo Dental College, \\ 2-9-18 Kanda-Misakicho, Chiyoda-ku, Tokyo 101-0061, Japan \\ 2) Department of Endodontics, Tokyo Dental College, \\ 2-9-18 Kanda-Misakicho, Chiyoda-ku, Tokyo 101-0061, Japan \\ 3) Tokyo Dental Junior College, \\ 2-9-18 Kanda-Misakicho, Chiyoda-ku, Tokyo 101-0061, Japan
}

Received 14 July, 2020/Accepted for publication 2 November, 2020 Published Online in J-STAGE 14 May, 2021

\begin{abstract}
We report a case of fixed prosthetic treatment for poor esthetics due to the position of the maxillary left lateral incisor in a 43-year-old woman. Initial examination revealed no carious lesions, but the tooth axis of the maxillary right canine showed mesial inclination of approximately $15^{\circ}$. Orthodontic treatment was first proposed but was declined by the patient as they did not wish to undergo a prolonged period of therapy. Therefore, recovery by extraction of the maxillary right lateral incisor and prosthetic treatment was proposed as an alternative. The method to be used for application of a 3-unit fixed partial denture and implant treatment was explained to the patient. She refused to give consent to this plan as well, however, due to concerns regarding the need to cut a lot from a nonproblematic tooth and the length of time such treatment would require. Therefore, the problem was finally treated by application of a cantilever single-retainer fixed partial denture while giving sufficient consideration to extraction and occlusal contact. Lithium disilicate was used for the material of the prothesis. At 1 year after completion of treatment, no problem was observed with either the prosthetic appliance or the abutment teeth.
\end{abstract}

Key words: Full crown - Cantilever fixed partial denture-

Monolithic all-ceramic crown - Lithium disilicate

Introduction

Reports on cantilever 2-unit fixed partial dentures using one abutment in the anterior tooth and premolar regions have recently accumulated $^{1-3,6,7,9,12)}$. This option has grown in 
popularity in prosthetic treatment for the loss of multiple teeth due to its high success rate ${ }^{12)}$. Miura et al. reported that a favorable course was achieved with a porcelain-layered zirconia cantilever single-retainer fixed partial denture using the canine as the abutment tooth in a case of a lost lateral incisor in the anterior tooth position ${ }^{4,5}$. On the other hand, although a cantilever fixed partial denture has a retainer on only one side of the mesiodistal pontic, the presence of at least 2 abutment teeth is recommended for the loss of a single tooth $^{11)}$. According to a report published in $\operatorname{Japan}^{10)}$, cantilever fixed partial dentures are not usually recommended. They are only considered acceptable in a very limited number of cases, even if 2 or more abutment teeth are present.

When improving the esthetic appearance a vital anterior tooth, treatment with a layered zirconia all-ceramic restoration or lithium disilicate monolithic all-ceramic restoration may be appropriate. Lithium disilicate is excellent for this purpose due to its transparency, which gives it high potential in terms of esthetic improvement. However, close attention must be paid to occlusion if this material is used, as its fracture strength is lower than that of zirconia.

A lithium disilicate cantilever singleretainer fixed partial denture was selected in the present patient with a view to improving poor esthetics in the region of the maxillary left lateral incisor. The treatment resulted in high patient satisfaction. This treatment plan was based on careful examination of occlusion. Prior informed consent was received from the patient for extraction and other procedures, and publication of the case in a journal.

\section{Case presentation}

\section{Outline of patient}

Patient: A 43-year-old woman.

Chief complaint: Poor esthetics in maxillary lateral incisor region.

History of present problems: She had been
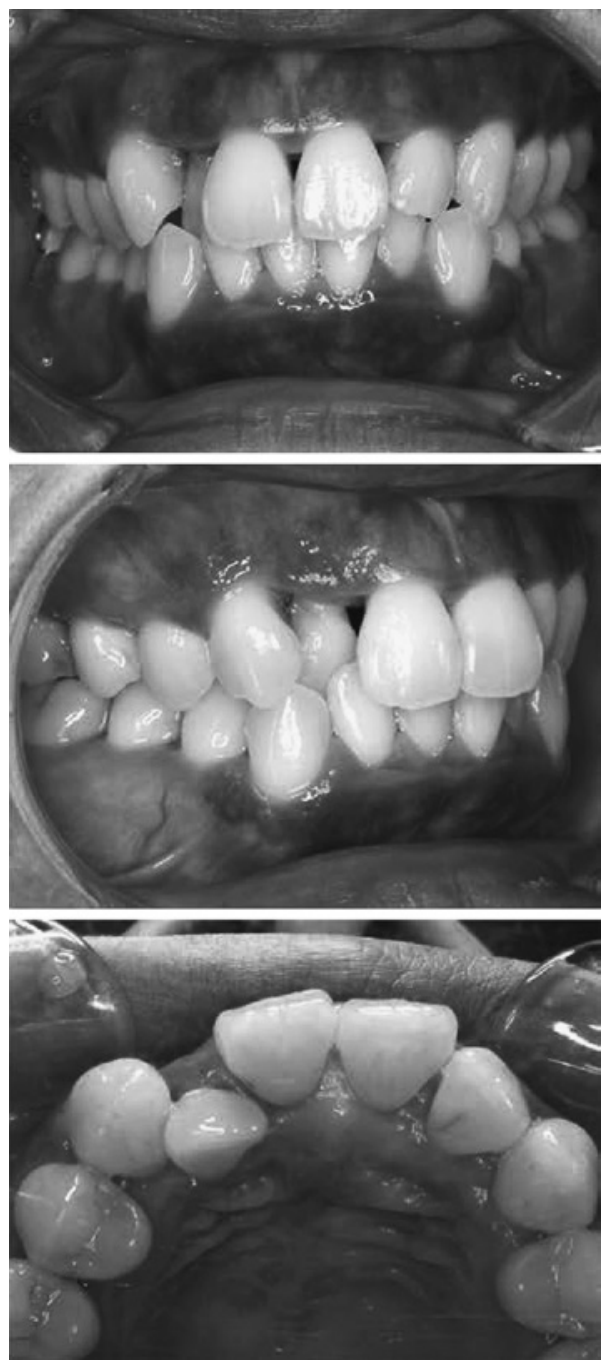

Fig. 1 Photographs of mouth at initiation of treatment (frontal, diagonally right protrusive, and occlusal views)

receiving treatment for moderate generalized chronic periodontitis since 2017 at a local clinic and the condition had improved. The patient later consulted her dentist regarding poor esthetics in the region of the maxillary right lateral incisor due to linguoversion. In December 2018, the patient was referred to the Department of Prosthodontics at our hospital for treatment (Fig. 1). Maintenance of periodontal treatment is also being continued 


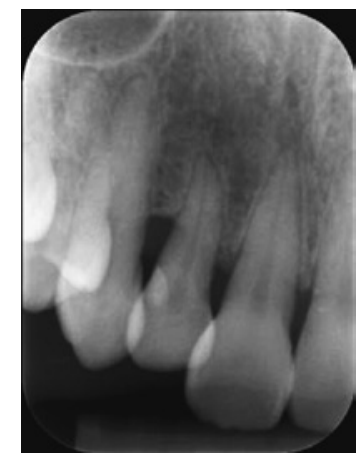

Fig. 2 Radiographic images at initiation of treatment

at another department here.

Present problems: All the anterior teeth were vital and symmetric. The tooth axis of the maxillary right canine showed mesial inclination of approximately $15^{\circ}$. Assessment of right lateral movement revealed an absence of guidance of occlusal contact by the maxillary canine.

No marked inflammatory finding was noted in the surrounding gingiva.

Results of the examinations: Periodontal tissue examination of the anterior tooth region revealed that the overall pocket depth was $3 \mathrm{~mm}$ or shallower, but that the crownroot ratio of the right lateral incisor was 1:0.8. Dental radiography revealed adsorption of alveolar bone at the center of the lateral incisor and canine mesial position. The current pathology was stable, however, and the caninecrown root ratio was normal (Fig. 2).

Diagnosis: Poor esthetics and masticatory dysfunction of the maxillary right lateral incisor due to linguoversion.

\section{Treatment plan}

The patient requested that treatment be as non-invasive as possible with minimal disturbance to current conditions. Orthodontic treatment was first proposed but was declined by the patient as they did not wish to undergo a prolonged period of therapy. Therefore, another treatment plan including extraction of the maxillary right lateral incisor and pros-

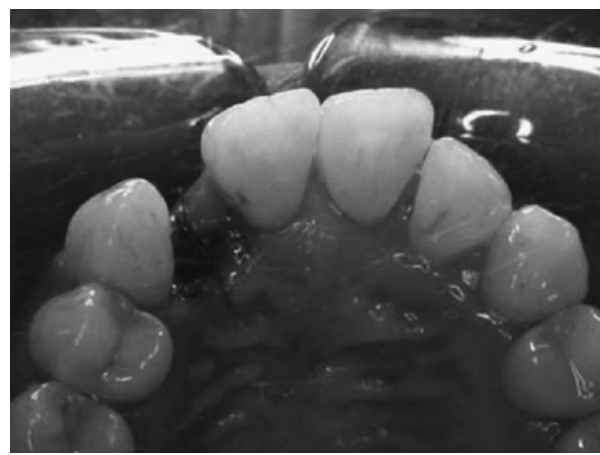

Fig. 3 Photograph of mouth after extraction of maxillary right lateral incisor

thetic treatment was proposed, although the level of invasiveness would be greater than that with orthodontic treatment. The method to be used for application of a 3-unit fixed partial denture and implant treatment was then explained to the patient. She refused to give consent to this plan as well, however, due to concerns regarding the need to cut a lot from a non-problematic tooth and the length of time such treatment would require.

Therefore, we finally proposed application of a mesial cantilever single-retainer fixed partial denture using the maxillary canine as an abutment, to which the patient gave her consent.

\section{Clinical Procedures and Outcomes}

\section{Content of treatment}

The maxillary right lateral incisor was extracted (Fig. 3). After achieving hemostasis of the wound by suturing, an artificial composite resin tooth (SURPASS, GC) adjusted to the morphology of a shell was applied to the bilateral adjacent teeth with 4-META/MMATBB resin (Superbond C\&B, Sun Medical) employing the brush-on technique (Fig. 4). Waxing-up was performed using the maxillary dental cast stored during the maintenance period while imaging the dentition to prepare provisional restoration of (3)2|. The stitches were removed at 1 week after extrac- 

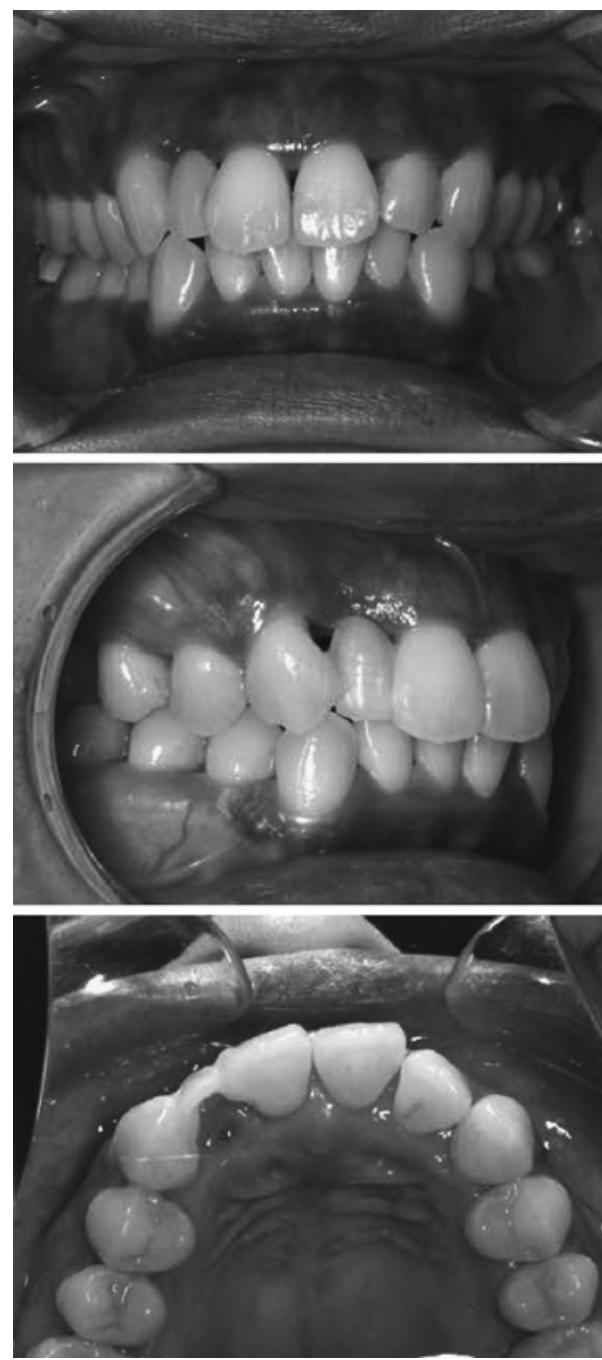

Fig. 4 Photographs of mouth after resin bonding of provisional crown (frontal, diagonally right protrusive, and occlusal views)

tion. After another month, the maxillary right canine abutment was prepared after confirming healing of the extraction socket (Fig. 5). Subsequently, a precise impression was taken using hydrophilic hybrid silicone rubber impression material (Fusion, wash-type and mono-phase type, GC) and the double-exclusion method. Photographs were taken for bite registration and color selection. The provisional restoration of (3) 2| was temporarily

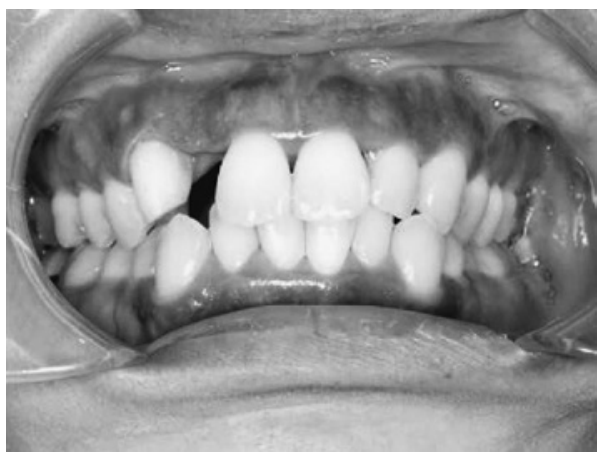

Fig. 5 Photographs of mouth after abutment formation

cemented (Hi-bond temporary cement, Shofu).

Lithium disilicate (e-MAX press, Ivoclar Vivadent Co.) was used for the cantilever single-retainer fixed partial denture, which was applied using the heat-press method (Fig. 6).

The denture was tried in the mouth to confirm fit, morphology, and pronunciation. The basement surface morphology of the pontic was the modified ridge lap type. Occlusion of the prosthetic appliance was set so as that contact would only be made with the canine in the central occlusal position, with no contact occurring during protrusive or right lateral movements. This was done in consideration of the occlusion pattern, which was characterized by right lateral movement as a group function of only the premolar and molar, which were natural teeth. For wearing, an adhesive primer for ceramics compounded with $\gamma$-MPTS and phosphate ester monomer (ceramic primer, Shofu) was applied after sandblasting of the inner surface of the crown. A composite adhesive resin cement with a universal color (Resicem, Shofu) was used (Fig. 7) was used as the setting material.

\section{Treatment course}

The course transited to maintenance after completion of treatment in March 2019. Examination of occlusion and the condition of the periodontal tissue, professional tooth maintenance, and oral hygiene instruction 

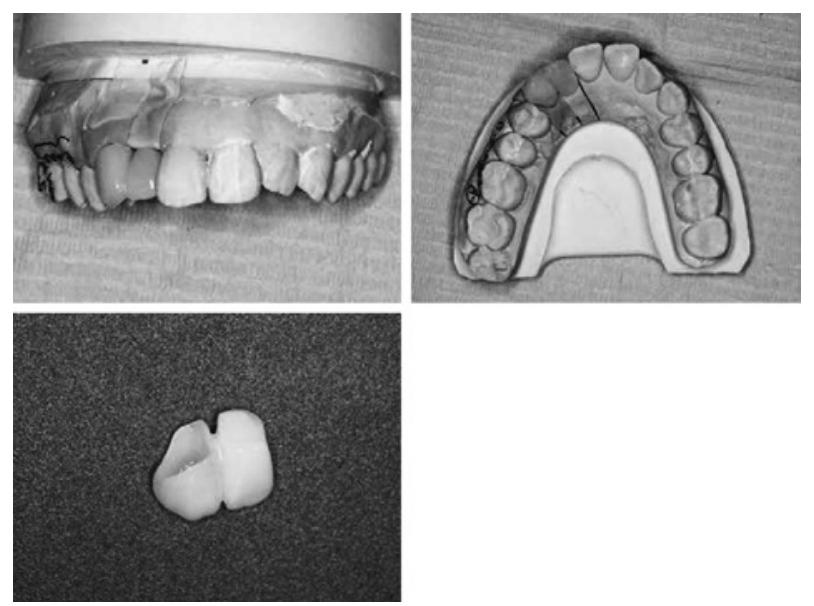

Fig. 6 Cantilever single-retainer-fixed partial denture

were performed every 4 months. At 1 year after completion of treatment, no problem with the prosthetic appliance had occurred, and no caries was observed in the abutment tooth. In addition, no change was observed in the state of occlusal contact, and the periodontal tissue showed a probing depth of 2-3 mm throughout the jaw. Overall, conditions were favorable and the patient expressed a high degree of satisfaction with the treatment she had received (Fig. 8).

\section{Discussion}

A lithium disilicate cantilever singleretainer fixed partial denture was selected in the present patient as a means of improving compromised esthetics due to linguoversion in the region of the maxillary lateral incisor.

A full-coverage-type metal-free fixed partial denture and adhesion fixed partial denture were considered applicable. The bilateral adjacent teeth were vital and free from caries, and the patient requested that treatment include as little drilling as possible. Conventionally in Japan ${ }^{10)}, 2$ or more abutment teeth are required in such cases. In this patient, however, the lost position was the lateral incisor, so it was easy to design a denture which did not apply high functional pressure, even though that region would host the pontic. Therefore, it was judged that a cantilever single-retainer fixed partial denture was indicated.

The maxillary canine has a larger root surface area and bearing capacity than the central incisor. The color and morphology of the bilateral central incisors were highly symmetric, suggesting that reproduction of color and morphology in the prosthetic would be very difficult. Shillingburg et al. mentioned that a single retainer could be used for the loss of a lateral incisor only when the adjacent canine to be used as an abutment had a long root and the surrounding bone was favorable ${ }^{8)}$. Therefore, in the present patient, treatment with a single retainer not using the central incisor as an abutment tooth but using the canine instead was considered optimal.

The canine occupied a relatively low position in the maxilla and failed to provide directional guidance when the patient was instructed to perform tooth grinding to elicit rightward lateral movement. However, wear due to occlusal contact was observed in the cutting edge of the canine, suggesting that occlusal contact had been taking place, possibly through bruxism during sleep. For the occlusal pattern of right lateral movement, 

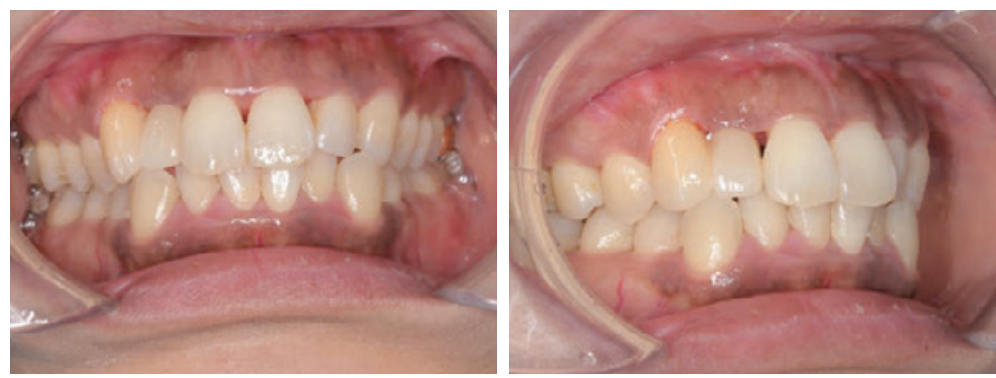

Fig. 7 Photographs of mouth after inserting prosthesis
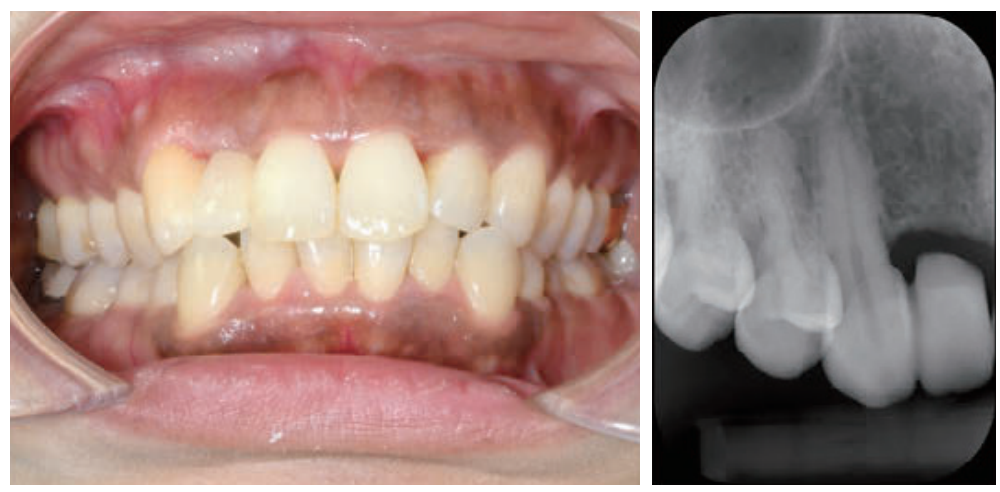

Fig. 8 Photographic and radiographic image of mouth after bridge worn for 1 year

group function was therefore selected and designed as to prevent contact, even in bruxism.

According to a recent review of cantilever fixed partial dentures, when the abutment was a vital tooth, the incidence of complications was lower and the survival rate higher than with a pulpless tooth ${ }^{7}$. However, it has been reported that pulp disease (pulpal death) was the most frequent complication in the vital tooth with the full coverage type of crown $^{1)}$, suggesting that the amount of preparation used determines the frequency of postoperative pulpal death. Generally, the abutment of a full coverage-type cantilever fixed partial denture is prepared similarly to that of a crown. In the present patient, the crownroot ratio of the canine used as an abutment tooth was favorable. Secondary caries devel- ops often with cantilever fixed partial dentures $^{2}$. Therefore, careful postoperative management is important in preventing secondary caries.

Some difficulty was encountered in the present patient. The tooth axis of the canine showed an inclination of $15^{\circ}$ against a line vertical to the occlusal plane, so the abutment was prepared under conditions of a slight mesial inclination in consideration of the dental pulp. This resulted in a large influence on the distal morphology of the central incisor in the wearing direction of the prosthesis. This could not be noticed in the provisional crown with artificial teeth adhered to the bilateral adjacent teeth until trying the full coverage provisional crown after abutment preparation. This suggests the need to perform careful examinations in establishing the 
conditions on which a treatment plan is to be based.

In achieving a successful cantilever fixed partial denture using a single retainer, it is of paramount importance to acquire specific information. This includes information on the appropriate selection to be made based on indications, procedures to be used for abutment preparation and adhesion, and materials to be used, all the while making sure they best match the techniques to be employed. Long-term accumulation of clinical data is necessary in achieving this.

Wear of the prosthetic appliance by bruxism was observed on follow-up and preparation of a sleep splint was considered.

\section{Conclusions}

The present patient required improvement of poor esthetics and correction of masticatory dysfunction due to linguoversion and low positioning of the maxillary right lateral incisor. This was achieved through minimallyinvasive treatment by applying a full coveragetype lithium disilicate cantilever singleretainer fixed partial denture for a missing tooth. A favorable outcome was achieved.

\section{Acknowledgements}

The authors wish to declare that no conflict of interest with regards to this report.

\section{References}

1) Chai J, Chu FC, Newsome PR, Chow TW (2005) Retrospective survival analysis of 3-unit fixed-fixed and 2-unit cantilevered fixed partial dentures. J Oral Rehabil 32:759-765.

2) Decock V, De Nayer K, De Boever JA, Dent M (1996) 18-year longitudinal study of cantilevered fixed restorations. Int J Prosthodont 9: 331-340.

3) Komine F, Tomic M (2005) A single-retainer zirconium dioxide ceramic resin-bonded fixed partial denture for single tooth replacement: a clinical report. J Oral Sci 47:139-142.

4) Miura S (2016) All- ceramic crown restorations for anterior open bite: A case report. Shika Shinbi 28:82-86. (in Japanese)

5) Miura $S$, Kasahara $S$, Yamauchi $S$, Fujisawa $M$ (2020) Esthetic improvement with single retainer cantilever 2-unit fixed dental prosthesis: A case report. Shika Shinbi 32:142-146. (in Japanese)

6) Mourshed B, Samran A, Alfagih A, Samaran A, Abdulrab S, Kern M (2018) Anterior cantilever resin-bonded fixed dental prostheses: A review of the literature. J Prosthodont 27: 266-275

7) Sailer I, Bonani T, Brodbeck U, Hämmerle CH (2013) Retrospective clinical study of singleretainer cantilever anterior and posterior glass-ceramic resin-bonded fixed dental prostheses at a mean follow-up 6 years. Int $\mathrm{J}$ Prosthodont 26:443-450.

8) Shillingburg HT, Sather DA, Wilson EL, Cain JR, Mitchell DL, Blanco LJ, Kessler JC (2020) Chapter 7, Treatment planning for the replacement of missing teeth - cantilever fixed partial dentures; Fundamentals of fixed prosthodontics, 4th ed., pp.169-174, Quintessence Publishing Co, Inc., Chicago.

9) Sillam CE, Cetik S, Ha TH, Atash R (2018) Influence of the amount of tooth surface preparation on the shear bond strength of zirconia cantilever single-retainer resinbonded fixed partial denture. J Adv Prosthodont 10:286-290.

10) Uchiyama $Y$ (1994) Indication and design of bridge. Nihon Hotetsu Shika Gakkai Zasshi 38:929-936. (in Japanese)

11) Wright WE (1986) Success with the cantilever fixed partial denture. J Prosthet Dent 55: $537-539$.

12) Yatani H (2019) The survival and complication rates of cantilever bridges using metal flame: A review of the literature. Nihon Hotetsu Shika Gakkaishi 11:193-205. (in Japanese)

\section{Correspondence:}

Dr. Syuntaro Nomoto

Department of Fixed Prosthodontics,

Tokyo Dental College,

2-9-18 Kanda-Misakicho, Chiyoda-ku,

Tokyo 101-0061, Japan

E-mail: nomotos@tdc.ac.jp 